\title{
Contrary to popular belief, ventilator- associated lower respiratory tract infections are less common in immunocompromised patients
}

\author{
Lieuwe D. Bos (10) ${ }^{1,2}$ \\ Affiliations: ${ }^{1}$ Dept of Respiratory Medicine, Academic Medical Center Amsterdam, Amsterdam, \\ The Netherlands. ${ }^{2}$ Intensive Care, Academic Medical Center Amsterdam, Amsterdam, The Netherlands. \\ Correspondence: Lieuwe D. Bos, Academic Medical Center Amsterdam, University of Amsterdam, Intensive \\ Care, Meibergdreef 9, M0-127, Amsterdam, 1105 AZ, The Netherlands. E-mail: l.d.bosdaamc.uva.nl
}

@ERSpublications

Ventilator-associated lower respiratory tract infections (VA-LRTIs) are less common in immunocompromised patients than in general ICU patients, so host response might be less important than anticipated in the pathophysiology of VA-LRTIs http://ow.ly/1V6Y30ih8yS

Cite this article as: Bos LD. Contrary to popular belief, ventilator-associated lower respiratory tract infections are less common in immunocompromised patients. Eur Respir J 2018; 51: 1800228 [https://doi. org/10.1183/13993003.00228-2018].

In this issue of the European Respiratory Journal, Moreau et al. [1] describe the impact of immunosuppression on incidence, aetiology and outcome of ventilator-associated lower respiratory tract infections (VA-LRTIs). The authors report a lower incidence of VA-LRTIs in immunosuppressed patients, with an odds ratio of 0.64 , which is in contradiction to their hypothesis and common belief.

It seems that the presumed positive association between immunosuppression and the development of VA-LRTI is mostly based on "common sense" and not on empirical data; no large study has previously quantified this relationship reliably. In their prospective observational cohort of almost 3000 intensive care unit (ICU) patients at risk for VA-LRTI, 22\% were defined as "immunosuppressed" due to the presence of a solid malignancy, haematological malignancy, AIDS or a history of allogeneic stem cell transplant, the use of immunosuppressive drugs or status after organ transplant. VA-LRTI was defined according to the currently accepted definitions [2]. Since immunosuppressed patients have an increased risk of decease during their stay on the ICU, and a deceased patient cannot develop VA-LRTI, competing risk analysis is necessary to quantify the risk of infection, and MOREAU et al. [1] adequately dealt with this type of bias [3-5]. They are to be applauded for their meticulous methodological approach. Thus, based on the available empirical evidence, we must accept that an immunosuppressed state does not increase the risk of VA-LRTI but might actually protect against VA-LRTI.

Received: Feb 012018 | Accepted: Feb 012018

Conflict of interest: L.D. Bos reports grants from the Dutch Lung Foundation (Young Investigator grant and PublicPrivate Partnership grant) and from the European Respiratory Society (short-term fellowship), and personal fees from Bayer (for consultancy), outside the submitted work.

Support statement: L.D. Bos is supported through the Dutch Lung Foundation (Longfonds) via a Young Investigator grant and a Public-Private Partnership grant (in association with Health Holland). He is also supported through a European Commission FP-7 Industry-Academia Partnerships and Pathways (IAPP) grant for the BreathDx consortium. Funding information for this article has been deposited with the Crossref Funder Registry.

Copyright OERS 2018 
Why did we believe the contrary and what are the consequences of these findings? Lower respiratory tract infections are the result of immigration of pathogenic bacteria into the lungs (figure 1). In ventilated patients, immigration into the lungs is increased through the presence of the endotracheal tube, positive pressure ventilation and an increased concentration of potential pathogenic bacteria in the stomach and oro-pharynx $[6,8]$. At the same time, elimination is decreased due to a reduced cough reflex and mucociliary clearance. As the host response is also an important factor in elimination of bacteria [7], especially in the absence of physical clearance of bacteria from the lower respiratory tract, it makes perfect pathophysiological sense that immunosuppressed patients more frequently develop VA-LRTIs. In fact, it was such a no-brainer that no one dared to question it.

\section{Can we now conclude that impaired host response does not contribute to the pathogenesis of VA-LRTI?}

Immunosuppression was broadly defined in the current study by MOREAU et al. [1] and was indicative of a general inability of the patient to produce a certain type of immune response. This mix of immunosuppressed states has been accepted as a clinical entity and has been shown to be important in the response to several types of pathogens, such as Pneumocystis jirovecii, Aspergillus, Histoplasma, Blastomyces, Coccidioides, Cryptococcus and Candida $[9,10]$. These fungi are not common pathogens in VA-LRTI and there is considerably less evidence that broadly defined immunosuppression contributes to the development of bacterial pneumonia. There is, however, abundant evidence that those immunosuppressed patients who do develop bacterial pneumonia have a high risk of mortality [11], and this finding is also supported by the data provided by MOREAU et al. [1].

For common respiratory pathogens (rather than opportunistic infections), local growth factors might be more important for the development of lower respiratory tract infections and these are not captured through the used definition of immunosuppression. Chronic obstructive pulmonary disease (COPD) and acute respiratory distress syndrome are well-established risk factors for ventilator-associated pneumonia (VAP) and are both associated with functional, anatomical and biochemical heterogeneity in the lung [6, $12,13]$. Local rather than systemic host response is altered in these patient groups. Based on the currently available data we can translate this as follows: opportunistic micro-organisms are omnipresent in the normal lung [14], therefore immigration and regional growth differences are less important and host response is the only factor keeping these micro-organisms from causing pneumonia. Conversely, common respiratory pathogens are fully capable of causing pneumonia, even with a completely functional immune response, and other factors are much more important in this case, eliminating immunosuppression as a risk factor. A conceptual framework for the development of VA-LRTIs is shown in figure 2.

But why would immunosuppression protect against VA-LRTI? MOREAU et al. [1] provide two potential explanations. It could be argued that immunosuppressed patients less frequently develop clinical characteristics of respiratory infections and therefore less commonly fulfil the criteria for VA-LRTI. As the

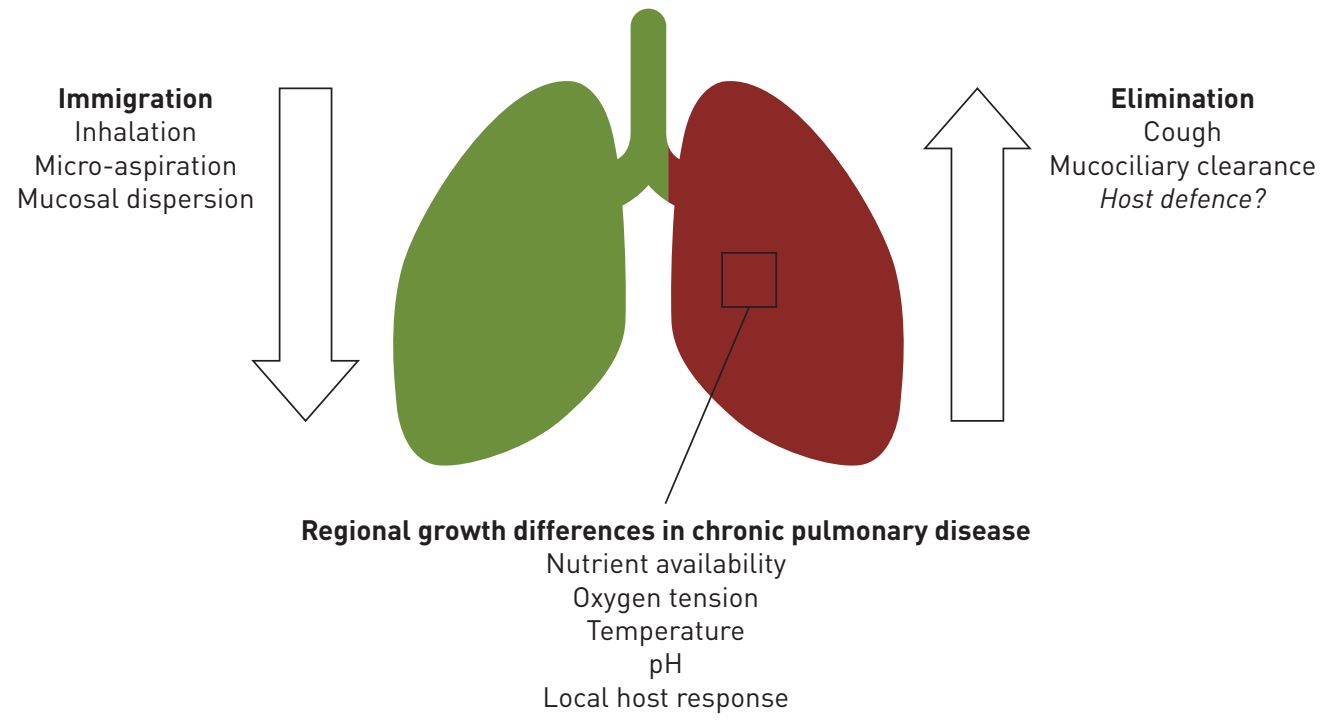

FIGURE 1 Factors influencing the pulmonary microbiome. Reproduced and modified from [6] with permission, and based on a personal interpretation of previously published figures, for example [7]. 
FIGURE 2 A conceptual framework for the development of ventilatorassociated lower respiratory tract infections. Blue shading indicates the steps where immunosuppression could be of importance. PPO: potential pathogenic organisms.

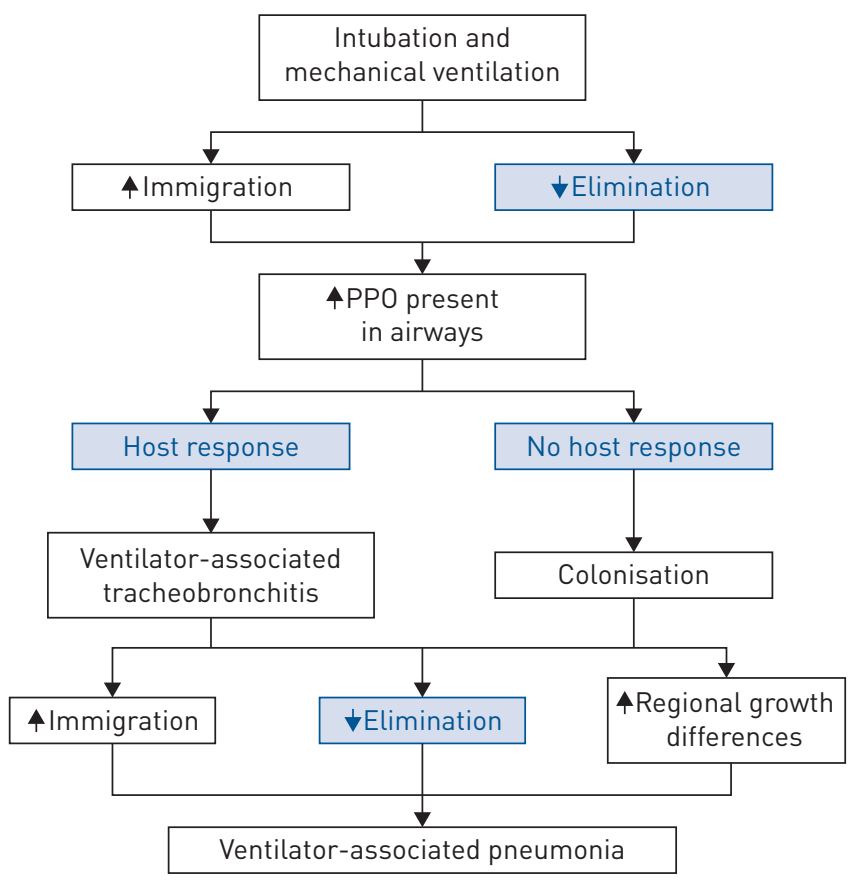

infection would have to be more severe for the same clinical characteristics to occur, this would also (partly) explain the higher mortality for immunosuppressed patients who do fulfil the criteria. However, only nine patients were neutropenic and the others ought to have been able to show signs of an inflammatory response. Pre-emptive use of antibiotics might be an alternative explanation. Immunosuppressed patients might receive broad-spectrum antibiotics earlier and might therefore be protected from the development of VA-LRTI. Administration of prophylactic antibiotics is associated with a lower incidence of VA-LRTI in other studies [15], and the addition of intravenous cephalosporine treatment to decontamination of the oro-pharynx/digestive tract is associated with protection against VAP [16]. These explanations are based on indirect evidence and are therefore not completely satisfactory. It remains to be determined if there are other mechanisms that can explain the observed protective effects.

Ventilator-associated tracheobronchitis (VAT) and VAP showed similar trends in their association with immunosuppression. VAT and VAP show the same clinical characteristics, such as fever, purulent sputum and inflammatory immune response [17, 18], and should both be prevented by broad-spectrum antibiotic treatment, so there is less concern about the aforementioned biases when comparing VAT and VAP prevalence. This is of particular interest as VAT is sometimes considered a precursor for VAP, progression of which depends on the host response [19]. However, the data from the TAVeM study reported by MoREAU et al. [1] suggest that immunosuppression is not an important indicator for progression of VAT to VAP. It might be that specific immunological mechanisms of importance are not affected in all immunocompromised patients [1]. One of the few studies on this topic suggests that complement activation is an important mechanism in the containment of VAT and this part of the innate response is little influenced by most of the studied immunosuppressed states [20]. However, there is a strong need for more evidence on the immunological and biochemical mechanisms that determine the progression towards pneumonia.

The study by Moreau et al. [1] does not close the book on the association between immunosuppression and the development of lower respiratory tract infections, but opens it. Studies like this exemplify how little we know about the risk factors and pathophysiology of respiratory diseases as common and as well studied as lower respiratory tract infections [7, 21]. Longitudinal analysis of patients developing VA-LRTIs might shed more light on this in the near future, as it has done for the development of exacerbations in cystic fibrosis [22] and COPD [23]. A combination of analysis of the microbiome [24, 25], the metabolic environment [26-28] and inflammatory markers [29] might provide insight into the host-pathogen interactions that are required for the development of VA-LRTIs and will provide more details on the exact immunological mechanisms that do play a role [30].

\section{References}

1 Moreau A-S, Martin-Loeches I, Povoa P, et al. Impact of immunosuppression on incidence, aetiology and outcome of ventilator-associated lower respiratory tract infections. Eur Respir J 2018; 51: 1701656. 
2 American Thoracic Society, Infectious Diseases Society of America. Guidelines for the management of adults with hospital-acquired, ventilator-associated, and healthcare-associated pneumonia. Am J Respir Crit Care Med 2005; 171: 388-416.

3 Noordzij M, Leffondré K, van Stralen KJ, et al. When do we need competing risks methods for survival analysis in nephrology? Nephrol Dial Transplant 2013; 28: 2670-2677.

4 Fine JP, Gray RJ. A proportional hazards model for the subdistribution of a competing risk. J Am Stat Assoc 1999; 94: 496-509.

5 Schoenfeld D. Survival methods, including those using competing risk analysis, are not appropriate for intensive care unit outcome studies. Crit Care 2006; 10: 103.

6 Roux D, van Oort PM, Ricard JD, et al. Airway microbiome research: a modern perspective on surveillance cultures? Ann Transl Med 2017; 5: 445.

7 Dickson RP, Erb-Downward JR, Huffnagle GB, et al. Towards an ecology of the lung: new conceptual models of pulmonary microbiology and pneumonia pathogenesis. Lancet Respir Med 2014; 2: 238-246.

8 Dickson RP. The microbiome and critical illness. Lancet Respir Med 2016; 4: 59-72.

9 Letourneau AR, Issa NC, Baden LR. Pneumonia in the immunocompromised host. Curr Opin Pulm Med 2014; 20: $272-279$.

10 Limper AH, Knox KS, Sarosi GA, et al. An official American Thoracic Society statement: treatment of fungal infections in adult pulmonary and critical care patients. Am J Respir Crit Care Med 2011; 183: 96-128.

11 Sousa D, Justo I, Domínguez A, et al. Community-acquired pneumonia in immunocompromised older patients: incidence, causative organisms and outcome. Clin Microbiol Infect 2013; 19: 187-192.

12 Rouzé A, Cottereau A, Nseir S. Chronic obstructive pulmonary disease and the risk for ventilator-associated pneumonia. Curr Opin Crit Care 2014; 20: 525-531.

13 Forel JM, Voillet F, Pulina D, et al. Ventilator-associated pneumonia and ICU mortality in severe ARDS patients ventilated according to a lung-protective strategy. Crit Care 2012; 16: R65.

14 Nguyen LD, Viscogliosi E, Delhaes L. The lung mycobiome: an emerging field of the human respiratory microbiome. Front Microbiol 2015; 6: 89.

15 Acquarolo A, Urli T, Perone G, et al. Antibiotic prophylaxis of early onset pneumonia in critically ill comatose patients. A randomized study. Intensive Care Med 2005; 31: 510-516.

16 Bos LD, Stips C, Schouten LR, et al. Selective decontamination of the digestive tract halves the prevalence of ventilator-associated pneumonia compared to selective oral decontamination. Intensive Care Med 2017; 43: 1535-1537.

17 Martin-Loeches I, Povoa P, Rodriguez A, et al. Incidence and prognosis of ventilator-associated tracheobronchitis (TAVeM): a multicentre, prospective, observational study. Lancet Respir Med 2015; 3: 859-868.

18 Craven DE, Hjalmarson KI. Ventilator-associated tracheobronchitis and pneumonia: thinking outside the box. Clin Infect Dis 2010; 51: Suppl. 1, S59-S66.

19 Nseir S, Povoa P, Salluh J, et al. Is there a continuum between ventilator-associated tracheobronchitis and ventilator-associated pneumonia? Intensive Care Med 2016; 42: 1190-1192.

20 Martin-Loeches I, Papiol E, Almansa R, et al. Intubated patients developing tracheobronchitis or pneumonia have distinctive complement system gene expression signatures in the pre-infection period: a pilot study. Med Intensiva 2012; 36: 257-263.

21 Nair GB, Niederman MS. Ventilator-associated pneumonia: present understanding and ongoing debates. Intensive Care Med 2015; 41: 34-48.

22 Gattinoni L, Bombino M, Pelosi P, et al. Lung structure and function in different stages of severe adult respiratory distress syndrome. JAMA 1994; 271: 1772-1779.

23 Wang Z, Bafadhel M, Haldar K, et al. Lung microbiome dynamics in COPD exacerbations. Eur Respir J 2016; 47: 1082-1092.

24 Zakharkina T, Martin-Loeches I, Matamoros S, et al. The dynamics of the pulmonary microbiome during mechanical ventilation in the intensive care unit and the association with occurrence of pneumonia. Thorax 2017; 72: 803-810.

25 Kelly BJ, Imai I, Bittinger K, et al. Composition and dynamics of the respiratory tract microbiome in intubated patients. Microbiome 2016; 4: 7.

26 van Oort PM, de Bruin S, Weda $\mathrm{H}$, et al. Exhaled breath metabolomics for the diagnosis of pneumonia in intubated and mechanically-ventilated intensive care unit (ICU)-patients. Int J Mol Sci 2017; 18: E449.

27 Schnabel R, Fijten R, Smolinska A, et al. Analysis of volatile organic compounds in exhaled breath to diagnose ventilator-associated pneumonia. Sci Rep 2015; 5: 17179.

28 van Oort PM, Povoa P, Schnabel R, et al. The potential role of exhaled breath analysis in the diagnostic process of pneumonia - a systematic review. J Breath Res 2018; 12: 024001.

29 Conway Morris A, Kefala K, Wilkinson TS, et al. Diagnostic importance of pulmonary interleukin-1 $\beta$ and interleukin-8 in ventilator-associated pneumonia. Thorax 2010; 65: 201-207.

30 Auffray C, Adcock IM, Chung KF, et al. An integrative systems biology approach to understanding pulmonary diseases. Chest 2010; 137: 1410-1416. 